ARTIGO

ARTICLE

\section{Causas múltiplas de mortalidade materna relacionada ao aborto no Estado de Minas Gerais, Brasil, 2000-2011}

\author{
Multiple causes of maternal mortality related to \\ abortion in Minas Gerais State, Brazil, 2000-2011
}

\section{Causas múltiples de mortalidad materna relacionada con el aborto, en el Estado de Minas Gerais, Brasil, 2000-2011}

\section{Resumo}

Este estudo objetivou analisar a evolução da mortalidade materna por aborto em Minas Gerais, Brasil, no período de 2000 a 2011, sob o enfoque das causas múltiplas de morte. Estudou-se as características sociodemográficas das mulheres, ano, local e causas básica e associada de óbito. Foi calculada a razão de morte materna (RMM) geral e específica por aborto em cada ano e o coeficiente de correlação de Spearman $(p<0,05)$, para avaliar a evolução das razões no período. Foram registrados 183 óbitos por aborto, 15\% dos óbitos maternos, e a RMM por aborto manteve-se estável. A razão causa múltipla e causa básica de óbito por aborto foi de 1,38. O uso do método de análise de causas múltiplas mostrou-se eficaz para dar maior visibilidade ao aborto. Os problemas oriundos da ilegalidade da prática de abortos favorecem o aparecimento de causas que mascaram mortes maternas e a sua subnotificação. Assim, ações intersetoriais são necessárias para a definição de estratégias, a fim de reduzir as desigualdades sociais e melhorar a qualidade dos serviços de atenção à mulher.

Mortalidade Materna; Aborto; Causas Múltiplas de Morte
Eunice Francisca Martins 1

Pollyanna Ferraz Botelho de Almeida 1

Cilene de Oliveira Paixão 1

Paula Gonçalves Bicalho 1

Livia de Souza Pancrácio de Errico 1

doi: 10.1590/0102-311X00133116

\author{
Correspondência \\ E. F. Martins \\ Escola de Enfermagem, Universidade Federal de Minas Gerais. \\ Av. Professor Alfredo Balena 190, Belo Horizonte, \\ MG 30130-100, Brasil. \\ eufram@yahoo.com.br \\ 1 Escola de Enfermagem, Universidade Federal de Minas Gerais, \\ Belo Horizonte, Brasil.
}




\section{Introdução}

A mortalidade materna é um problema de saúde pública mundial e uma prioridade global de saúde. Estima-se que 287.000 mortes maternas ocorreram em todo o mundo no ano de 20101 . Devido à magnitude do problema, a redução dessa mortalidade foi incluída na lista dos Objetivos de Desenvolvimento do Milênio definidos pela Organização das Nações Unidas (ONU). A meta era reduzir em 75\% a razão da mortalidade materna até 2015, no entanto, as estimativas globais sobre o declínio desta mortalidade revelam que os resultados atingidos não foram suficientes para alcançar a meta na maioria dos países 2,3 .

No caso do Brasil, para atingir a meta do quinto Objetivos de Desenvolvimento do Milênio, o país deveria apresentar uma razão de morte materna (RMM) igual ou inferior a 35 óbitos por 100 mil nascidos vivos até 2015 4. Em 2011, a RMM no Brasil foi igual a 64,8 óbitos por 100 mil nascidos vivos (Departamento de Informática do SUS. Rede interagencial de informações para a saúde. Indicadores e dados básicos para a saúde, 2012. http://tabnet.datasus.gov.br/cgi/idb2012/C03b.htm, acessado em $31 / \mathrm{Jul} / 2015)$, que mostra o desafio para atingir a meta proposta. Diante desse quadro, é imperioso que haja melhor compreensão dos fatores determinantes e revisão das estratégias de ação 4 .

Dessa forma, o estudo das causas de morte materna tem o potencial de contribuir na definição dessas novas estratégias. Observa-se que as principais causas são consequentes à hemorragia, hipertensão, sepse, aborto e embolismo 1,5. O desafio para a redução da mortalidade materna por aborto é ainda maior, tendo em vista situações como a clandestinidade e a ilegalidade 6 . A Organização Mundial da Saúde (OMS) estima que em 2008 cerca de 13\% das mortes maternas em todo o mundo, equivalente a 47 mil, foram devido a abortos inseguros 7 . No Brasil, o aborto está entre as cinco principais causas de mortalidade materna, e relaciona-se a aproximadamente 5\% do total de óbitos maternos 8 . Por esse motivo, nos últimos anos, assiste-se no país a uma grande discussão sobre a descriminalização do aborto, envolvendo, sobretudo, um complexo conjunto de aspectos políticos, legais, morais, religiosos, sociais e culturais.

Vários estudos discutem que a ilegalidade do aborto induzido pouco coíbe a sua prática e traz consequências negativas para a saúde das mulheres, majoritariamente, as pobres, que não têm acesso aos recursos para o aborto seguro 9,10,11,12,13,14. Acrescentando à questão da ilegalidade, os aspectos culturais e religiosos inibem as mulheres de declararem seus abortamentos, o que contribui para a omissão do aborto como fator desencadeante dos eventos que levaram à morte ${ }^{9}$. Nesse contexto, a avaliação da magnitude da mortalidade materna também é comprometida. Observam-se falhas no preenchimento da Declaração de Óbito (DO) 15, subinformação e sub-registro das declarações das causas de óbitos 16 . Os problemas mais comuns são a ausência da informação sobre a existência de gravidez nos últimos doze meses nas DO das mulheres em idade fértil e a declaração apenas da causa terminal, sem considerar a sucessão de eventos que levou à morte. A causa de óbito oriunda desse processo caracteriza a chamada morte materna mascarada ou presumível. Dessa forma, oculta-se a causa da morte e impede-se a identificação do óbito materno 17.

Nos últimos anos, algumas medidas foram adotadas no sentido de minimizar os efeitos da subnotificação sobre a prevalência da mortalidade materna obtida por meio dos dados do Sistema de Informação sobre Mortalidade (SIM). Entre elas, destaca-se o processo de investigação realizada pelos comitês de prevenção da mortalidade materna, com vistas a identificar a relação entre a causa básica do óbito e uma possível gravidez ${ }^{18}$. Outra estratégia adotada é o método de pesquisa chamado Reproductive Age Mortality Study (RAMOS), desenvolvido para mensurar o número de mortes maternas subnotificadas e calcular um fator de ajuste para a correção dos dados oficiais, com base nos dados de mortes de mulheres em idade reprodutiva 19.

Considerando que os níveis de prevalência dos óbitos por aborto podem ser influenciados pela subnotificação, supõe-se que o estudo de causas múltiplas de morte, ou seja, o conjunto das causas básica e associada ao óbito, pode ser um método auxiliar para a correção desse problema. A abordagem de causas múltiplas de morte, ao evidenciar causas de morte que permaneceriam ocultas pelo enfoque tradicional, possibilita um melhor entendimento do conjunto de enfermidades que levam ao óbito 20 .

Dessa forma, este trabalho propõe analisar a evolução da mortalidade materna relacionada ao aborto em Minas Gerais, Brasil, no período de 2000-2011, com base nos dados do SIM, sob o enfoque das causas múltiplas de morte. 


\section{Metodologia}

Estudo ecológico de uma série temporal dos óbitos por abortamento no Estado de Minas Gerais, no período de 2000-2011. Este trabalho utilizou os dados do SIM, download em 23 de junho de 2014 do sítio do DATASUS (http://www.datasus.gov.br). O SIM; desde 1979, é o sistema oficial de informação sobre mortalidade no Brasil e usa a DO como documento padrão para o registro dos dados de mortalidade 17.

Este estudo utilizou o método de análise das causas múltiplas de morte, conforme conceituado por Santo 21. Causas múltiplas é a denominação dada ao conjunto formado pela causa básica e causas associadas. A causa associada é composta pelas causas consequenciais e contribuintes. As causas consequenciais são aquelas que compõem a cadeia de acontecimentos patológicos que levou ao óbito. Por sua vez, as causas contribuintes, apesar de não estarem associadas à patologia ou lesão que causou a morte, são condições mórbidas que contribuíram para o seu agravamento. As causas consequenciais estão descritas na Parte I da declaração de óbito, nas linhas anteriores à causa básica. Na parte II do atestado de óbito estão descritas as causas contribuintes 22. A esse conjunto, causa básica e causas associadas, Santo 21 atribuiu o nome de causas múltiplas. Assim, foram incluídos no estudo todos os óbitos de mulheres em idade fértil (10-49 anos), cuja causa básica e/ou associada de morte constasse o aborto, agrupamento O00-O08 do capítulo XV da Classificação Estatística Internacional de Problemas e Doenças Relacionados à Saúde - 10a Revisão (CID-10) 23.

As variáveis estudadas foram causas múltiplas (causa básica e associada) de morte, aquelas referentes às características sociodemográficas das mulheres (idade, escolaridade em anos, estado civil e raça/ cor), ano e local de ocorrência do óbito.

Calculou-se a RMM geral e específica por causas básicas. A RMM específica relacionada ao aborto foi distribuída segundo causa básica, causas associadas e causa múltipla ou total de suas menções. Realizou-se a distribuição proporcional dos óbitos maternos relacionados ao aborto segundo causa múltipla, causa básica e causa associada. Essa última foi classificada em causa básica materna e causa básica não materna. A terminologia causa básica materna foi usada para todos os óbitos cuja causa básica estava descrita nos agrupamentos do capítulo XV da CID-10 - Gravidez, parto e puerpério, com exceção daquelas descritas no agrupamento gravidez que termina em aborto (O00-O08). As causas básicas não maternas incluíram as demais causas descritas em outros capítulos da CID-10. Foi calculada a razão causa múltipla/causa básica para avaliar o incremento do número de mortes maternas relacionadas à gravidez terminada em aborto. Para avaliar a evolução da RMM no período foram calculados os Coeficientes de Correlação de Spearman, e o nível de significância considerado foi de 5\%. No cálculo da RMM usou-se o método direto conforme proposto pela Rede Interagencial de Informações para a Saúde. Nesse método, a RMM é calculada considerando-se o número de óbitos de mulheres residentes segundo causas, sobre o número de nascidos vivos de mães residentes, multiplicado por 100 mil 24. O número de nascidos vivos foi oriundo do Sistema de Informação sobre Nascidos Vivos (SINASC), também disponível online no sítio do DATASUS. Realizou-se a distribuição proporcional dos óbitos por aborto, segundo as variáveis sociodemográficas e local de ocorrência e o estudo descritivo da distribuição proporcional das causas múltiplas de óbitos.

O software IBM SPSS versão 21.0 (IBM Corp., Armonk, Estados Unidos) foi utilizado para a análise estatística.

Por serem os dados públicos, disponíveis on-line, sem identificação pessoal ou institucional, não houve necessidade de aprovação do estudo em Comitê de Ética de Pesquisa.

\section{Resultados}

No período de 2000-2011, foram registrados 82.790 óbitos de mulheres em idade fértil (10-49 anos) na população residente no Estado de Minas Gerais, destes 1.219 (1,47\%) tiveram morte materna como causa básica. A Figura 1 mostra a evolução da proporção das principais causas de morte materna para o período. A contribuição proporcional do aborto na composição do grupo de causas foi de cerca de $15 \%$, e não houve alterações expressivas no período. As demais causas apresentaram variações, destacando-se os transtornos hipertensivos e as complicações do trabalho de parto e do parto. 


\section{Figura 1}

Evolução da Razão de Morte Materna por causas segundo ano de ocorrência. Minas Gerais, Brasil, 2000-2011.

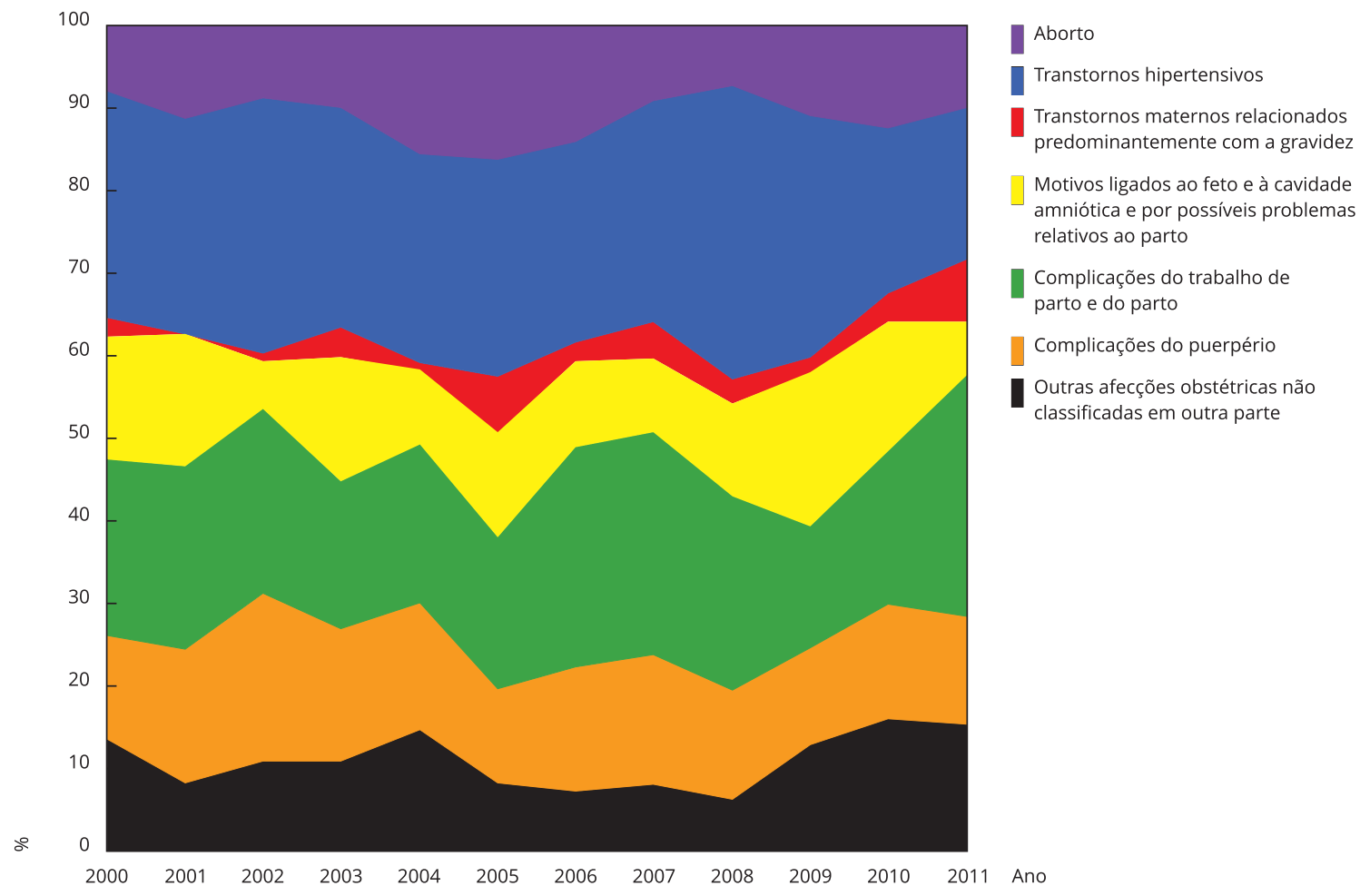

O aborto foi identificado como causa básica em 133 óbitos e como causa associada em outros 50, totalizando 183 mortes relacionadas ao aborto. Nos óbitos cuja causa associada estava relacionada ao aborto, as causa básicas foram classificadas em maternas e não maternas, 28 (56\%) e 22 (44\%), respectivamente (Figura 2).

O detalhamento da análise das mortes maternas por aborto mostrou que a principal causa básica foi o aborto não especificado (33,8\%), seguido da gravidez ectópica $(21,1 \%)$ e falha na tentativa de aborto (16,5\%). Nos óbitos maternos cuja causa básica pôde ser relacionada ao aborto destacaram-se as complicações do trabalho de parto e do parto $(28,6 \%)$ e complicações relacionadas predominantemente ao puerpério $(17,9 \%)$. Considerando os óbitos relacionados ao aborto, classificados na categoria causa básica não materna, sobressaíram aqueles relacionados às doenças do aparelho geniturinário $(22,7 \%)$ e à septicemia não especificada $(18,2 \%)$.

A caracterização dos óbitos relacionados ao aborto descrita na Tabela 1 evidencia que este foi mais prevalente em mulheres de 20-34 anos, solteiras (68\%) e negras (70,5\%), em sua maioria com menos de 7 anos de estudos. Vale ressaltar que em torno de $40 \%$ dos dados referentes à escolaridade e ao momento do óbito em relação à gravidez ou puerpério não estavam registrados no banco.

A Figura 3 mostra o aumento no nível da mortalidade materna por aborto quando esta é analisada pelo método de causas múltiplas. Assim, a razão entre as causas múltipla e básica de morte foi de 1,38, com incremento de $38 \%$ no período. Além disso, observa-se que ambas as curvas apresentam variações em seus níveis, sendo a menor razão em 2007 (3,5) e a maior em $2010(6,7)$.

A razão de mortalidade materna em Minas Gerais apresentou variações no período estudado, sendo a razão máxima de 46,6 no ano de 2010 e a mínima de 32,1 em 2005. A variabilidade nas razões de 


\section{Figura 2}

Óbitos maternos relacionados ao aborto por tipo de causas. Minas Gerais, Brasil, 2000-2011.

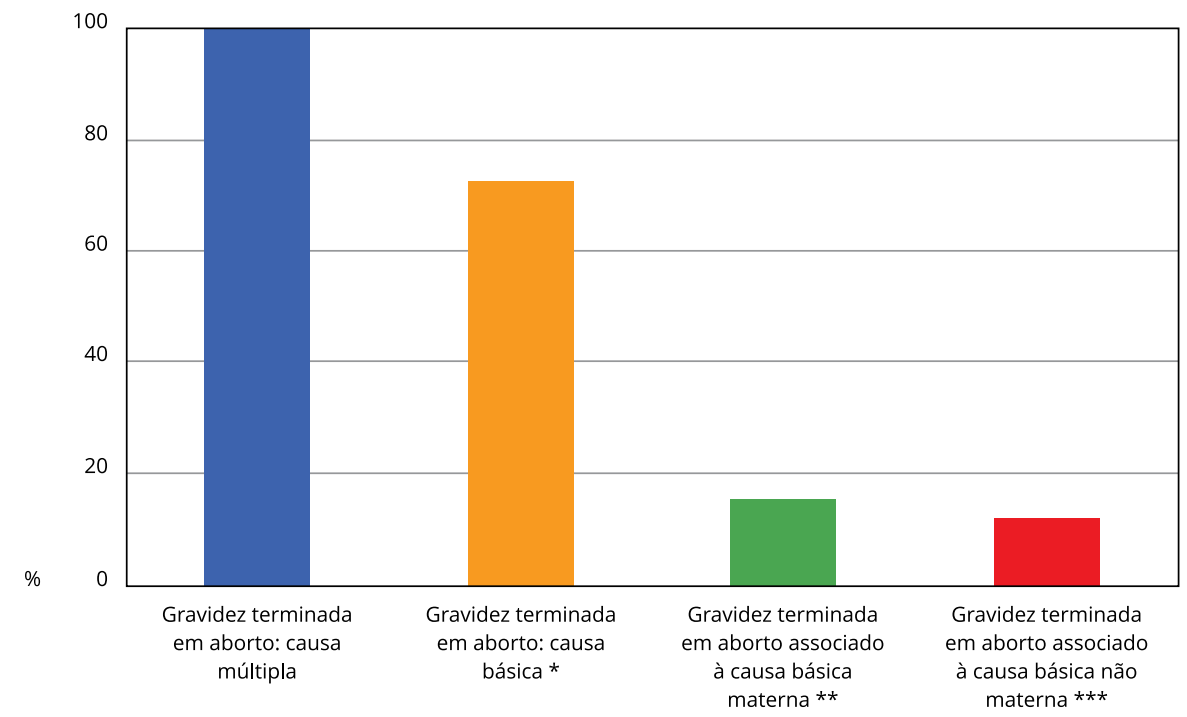

* 000-008: gravidez que termina em aborto;

** 010-016: edema, proteinúria, e transtornos hipertensivos na gravidez, no parto e no puerpério; O20-O29: outros transtornos maternos relacionados predominantemente com a gravidez; O30-048: assistência prestada à mãe por motivos ligados ao feto, à cavidade amniótica e problemas relativos ao parto; 060-075: complicações do trabalho de parto e do parto; 085-092: complicações relacionadas predominantemente com o puerpério; 094-099: outras afecções obstétricas não classificadas em outra parte; 000-008: gravidez que termina em aborto;

*** A30-A49: outras doenças bacterianas; C00-C97: neoplasias malignas; D55-D59: anemias hemolíticas; D60-D64: anemias aplásticas e outras anemias; D65-D69: defeitos de coagulação, púrpura e outras afecções hemorrágicas; I05-I09: doença reumática crônica do coração; I30-152: outras formas de doença do coração; I60-169: doenças cerebrovasculares; J20-J22: outras infecções agudas das vias aéreas inferiores; J80-J84: outras doenças respiratórias que afetam principalmente o interstício; K20-K31: doenças do esôfago, do estômago e do duodeno; K55-K63: outras doenças dos intestinos; K70-K77: doenças do fígado; N17-N19: insuficiência renal; N70-N77: doenças inflamatórias dos órgãos pélvicos femininos; N80-N98: transtornos não inflamatórios do trato genital feminino.

morte materna, morte materna relacionada ao aborto por causa múltipla e a participação proporcional das causas relacionadas ao aborto no total das causas de morte materna não apresentou diferenças estatisticamente significativas (Tabela 2).

\section{Discussão}

A análise da prevalência do aborto é dificultada pelo fato deste ser uma prática ilegal no Brasil, salvo em algumas situações especificadas pelo artigo 128 do Código Penal Brasileiro, promulgado em 194025. Esse fato vai implicar tanto a avaliação do nível ou da magnitude do evento quanto a sua caracterização. O status de ilegalidade, via de regra, induz as mulheres, por medo das consequências legais, a não procurarem os serviços de saúde ou o fazerem tardiamente, acentuando seus riscos e a gravidade do quadro 10,26. Dessa forma, pode ocorrer subnotificação nos registros oficiais, principalmente quando se trata dos abortos provocados. Além da subnotificação, a ilegalidade do procedimento implica, na 
Características da mulher e local de ocorrência dos óbitos relacionados ao aborto. Minas Gerais, Brasil, $2000-2011$.

\begin{tabular}{|c|c|c|}
\hline Características & $\mathbf{n}$ & $\%$ \\
\hline \multicolumn{3}{|l|}{ Idade materna (anos) } \\
\hline $14-19$ & 16 & 8,7 \\
\hline $20-34$ & 133 & 72,7 \\
\hline $35-46$ & 34 & 18,6 \\
\hline \multicolumn{3}{|l|}{ Escolaridade materna (anos) } \\
\hline Até 3 & 26 & 25,0 \\
\hline $4-7$ & 36 & 34,6 \\
\hline $8-11$ & 35 & 33,6 \\
\hline$\geq 12$ & 7 & 6,7 \\
\hline \multicolumn{3}{|l|}{ Estado civil } \\
\hline Solteira & 117 & 68,0 \\
\hline Casada & 43 & 25,0 \\
\hline Viúva & 3 & 1,7 \\
\hline Separada judicialmente & 9 & 5,2 \\
\hline \multicolumn{3}{|l|}{ Raça/Cor } \\
\hline Branca & 44 & 29,5 \\
\hline Negra & 105 & 70,5 \\
\hline \multicolumn{3}{|l|}{ Local de ocorrência } \\
\hline Hospital & 179 & 97,8 \\
\hline Domicílio & 3 & 1,6 \\
\hline Via pública & 1 & 0,5 \\
\hline Total & 183 & 100,0 \\
\hline
\end{tabular}

Nota: excluídos os casos ignorados.

Fonte: Departamento de Informática do SUS. Sistema de Informação sobre Mortalidade.

maioria das vezes, a prática do aborto inseguro. O aborto inseguro relaciona-se aos procedimentos para o término de uma gestação, realizado por pessoas não capacitadas e/ou em locais insalubres e sem segurança para o atendimento de eventuais complicações 10.

Os efeitos da ilegalidade da prática do aborto também irão implicar a compreensão do significado da invariabilidade do nível da prevalência das taxas de mortalidade por aborto ao longo do período do estudo 10. Essa invariabilidade tem duplo sentido, um relacionado às questões do registro da ocorrência do evento, como já mencionado, e outro ligado às limitações das ações direcionadas para a prevenção ou correção das eventuais complicações da prática do aborto, principalmente no caso do aborto inseguro.

Em contraponto, as variações na prevalência da mortalidade materna, considerando outras causas, podem ser imputadas às diversas ações realizadas para a melhoria da assistência à gestante, parturiente e puérpera. Por exemplo, a redução da mortalidade por causas relacionadas aos transtornos hipertensivos e ao trabalho de parto e parto tem sido objeto das políticas de atenção ao parto e nascimento, tanto no âmbito estadual quanto no federal 27,28,29,30,31. Dessa forma, a estabilidade na prevalência das mortes relacionadas ao aborto pode ser entendida como um marcador que sinaliza para a necessidade de definições de ações efetivas para a eliminação do aborto inseguro, visto que este é classificado como um evento evitável 32.

A evitabilidade do aborto identificada neste estudo sugere a existência de problemas relacionados ao diagnóstico precoce e oportuno. Além disso, a mortalidade por aborto enquadra-se dentro do grupo das iniquidades em saúde, uma vez que por meio de medidas ou tecnologias adequadas poderá ser reduzida 33 . Nessa perspectiva, a melhoria da assistência caminha pari passu com avanços na formação e atualização profissional, assim como na apropriada discussão dos estigmas da sociedade em relação à mulher em situação de abortamento 34 . 


\section{Figura 3}

Evolução da Razão de Morte Materna (RMM) por aborto por causa básica e razão de morte materna relacionada ao aborto por causa múltipla. Minas Gerais, Brasil, 2000-2011.

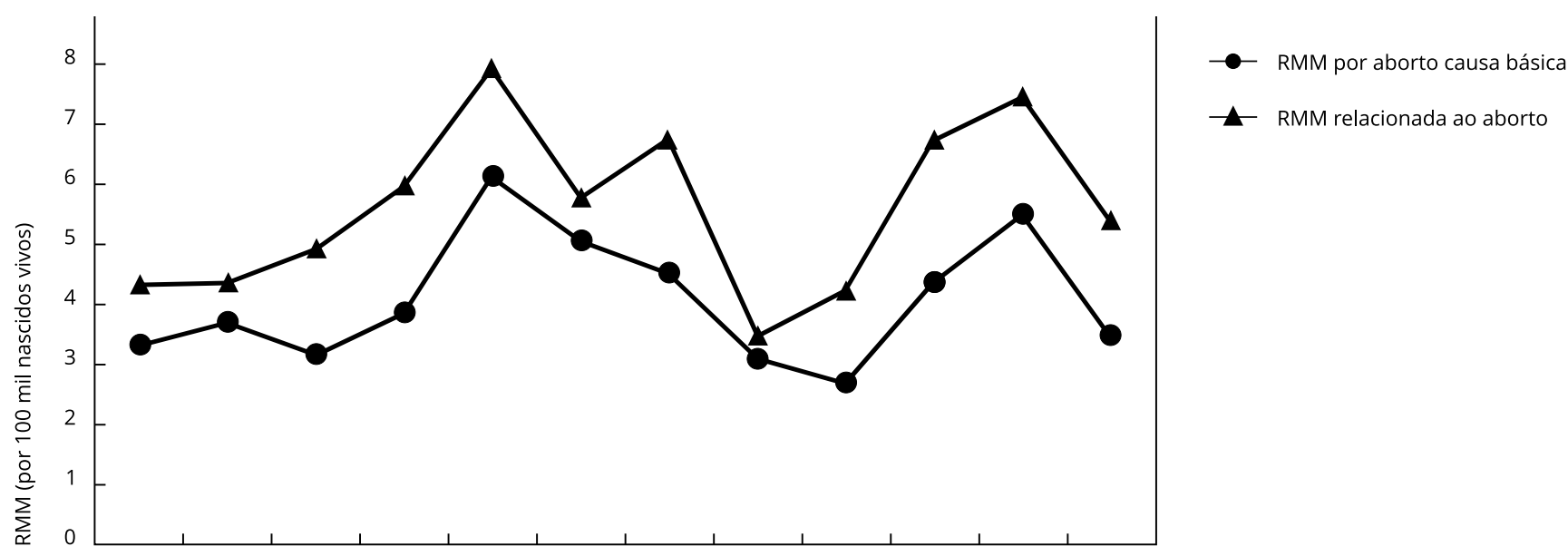

Tabela 2

Evolução da Razão de Mortalidade Materna (RMM), Razão de Morte Materna por Causas Múltiplas relacionada ao Aborto (RMMCM_A) e proporção da participação da Razão de Morte Materna por Causas Múltiplas relacionada ao Aborto em relação à Razão de Morte Materna. Minas Gerais, Brasil, 2000-2011.

\begin{tabular}{lccc}
\hline Ano & RMM & RMMCM_A & \% de RMMCM_A na RMM \\
\hline 2000 & 42,7 & 4,3 & 10 \\
2001 & 36,2 & 5,4 & 14,9 \\
Coeficiente de Correlação Spearman's rho & 0,08 & 0,22 & 0,32 \\
Valor de p & 0,8 & 0,48 & 0,32 \\
\hline
\end{tabular}

Fonte: Departamento de Informática do SUS. Sistema de Informação sobre Mortalidade.

A concentração dos óbitos nos grupos sociais menos afluentes, com predomínio de mulheres com escolaridade insuficiente e da raça/cor negra, sustenta os argumentos que situam o óbito por aborto como uma condição marcadora da iniquidade em saúde. Isso nos leva a reconhecer que para além da ilegalidade, outros fatores contribuem para que a mulher se submeta ou realize o aborto inseguro. Entre esses, destaca-se o contexto social de vida e trabalho dessas mulheres, que pode contribuir para mantê-las à margem de uma assistência à saúde de qualidade 26. A condição socioeconômica emerge como um dos fatores que compõem esse quadro, assim como descrito nos estudos de Cecatti et al. $35 \mathrm{e}$ Faria et al. 36. Vale lembrar que, historicamente, as mulheres negras apresentam maior vulnerabilidade quando se analisa o acesso a serviços para o atendimento das suas necessidades no âmbito dos direitos sexuais e reprodutivos. Diante disso, não é inesperado o fato de que entre elas está a maior prevalência de gravidez indesejada, aumentando a probabilidade de estas realizarem abortos inseguros 37 .

De fato, o que está em análise é o acesso e a utilização de serviços que desenvolvem ações no âmbito da saúde sexual e reprodutiva. Na reflexão sobre a eficácia da assistência à mulher em situação de aborto, observa-se que a prevenção é melhor alcançada por atuação competente na orientação e 
provisão de métodos contraceptivos adequados às necessidades de cada mulher. Vale lembrar que a mortalidade materna por aborto envolve aspectos que devem ser trabalhados e solucionados de forma intersetorial, visando às melhorias na escolaridade e no acesso aos serviços de saúde sexual e reprodutiva, principalmente para populações vulneráveis socialmente 18 .

As causas básicas de morte aborto não especificado e falha na tentativa do aborto são causas sentinelas uma vez que estas podem representar a ponta do iceberg formado pelos problemas oriundos da prática de abortos ilegais. Esses geralmente culminam com complicações e a busca tardia dos serviços de saúde pública. Na avaliação da OMS 26, as mulheres, ao se submeterem a um abortamento inseguro, não procuram atendimento médico pela dificuldade de reconhecer possíveis complicações, por carecerem dos meios econômicos necessários ou por temerem abuso, maltrato ou represália legal. No caso dos óbitos por gravidez ectópica, a discussão centra-se na capacidade dos serviços em realizar a captação precoce das gestantes 38 e de como as equipes assistenciais dos serviços de pronto atendimento valorizam os quadros de abdome agudo nas mulheres atendidas.

As causas básicas não maternas relacionadas ao aborto podem ser compreendidas como causas que mascaram a morte materna ou presumíveis de morte materna, isto é, a morte cuja causa básica do óbito relaciona-se ao ciclo gravídico-puerperal, porém isto não consta como causa básica na declaração de óbito 18 . Esse fato contribui para a subnotificação dos óbitos maternos e está relacionado ao preenchimento incorreto das declarações de óbito, em decorrência do desconhecimento dos médicos acerca do correto preenchimento da declaração de óbito e da relevância deste documento como fonte de dados de saúde. Assim, a ausência do registro da vinculação do óbito de mulher em idade fértil com as fases do ciclo gravídico puerperal representa um dos grandes problemas para a correta mensuração da prevalência da morte materna ${ }^{39}$. Dessa forma, o uso da metodologia das causas múltiplas representou um avanço na compreensão do problema, uma vez que ampliou o número de causas de óbitos associados ao aborto para além do capítulo XV da CID-10.

Uma vez que as causas não maternas aparecem como causa básica, sua associação com o aborto só foi possível porque a base do método das causas múltiplas fundamenta-se na análise dos demais campos de causas associadas ao óbito. A contribuição das causas que mascaram a morte materna para a mensuração da prevalência do aborto nos remete a processo semelhante que tem sido utilizado para redimensionar a magnitude da mortalidade materna geral ${ }^{17}$. Ressalta-se que a lista dessas causas que mascaram a morte materna pode ser ampliada pelo acréscimo de outras causas, tais como, anemias e neoplasias, o que poderá contribuir para redimensionar a magnitude da mortalidade materna.

O uso de dados secundários do SIM apresenta limitações relacionadas à completude dos dados.

\section{Conclusão}

A evolução da mortalidade materna relacionada ao aborto no Estado de Minas Gerais no período de 2000-2011 foi estável. O redimensionamento da magnitude da prevalência de morte relacionada ao aborto em função do método de análise de causas múltiplas mostrou-se eficaz para dar maior visibilidade ao problema no estado.

A ilegalidade da prática do aborto induz o preenchimento inadequado das declarações de óbitos, o que acarreta a subestimação da morte materna por esta causa. Esse problema não é resolvido apenas com o treinamento da equipe médica quanto ao preenchimento da declaração de óbito, ele exige soluções que articulam diferentes setores da nossa sociedade. Os serviços de saúde devem se implicar e serem implicados na definição de estratégias eficazes que garantam às mulheres condições de organizarem suas vidas no que diz respeito às questões da sua saúde sexual e reprodutiva. Entretanto, há de se chamar a atenção para a necessidade de que outros setores atuem concretamente na redução da vulnerabilidade social imbricada no cotidiano das mulheres das classes menos afluentes. Assim sendo, é fundamental o planejamento e desenvolvimento de ações intersetoriais que visem à redução das desigualdades sociais e a melhoria da qualidade dos serviços de atenção à mulher. Nesse contexto, ressalta-se a importância dos comitês de prevenção do óbito materno e a ampliação da capacidade de interlocução destes com outros setores da sociedade. 


\section{Colaboradores}

E. F. Martins participou da elaboração do projeto de pesquisa, organização, análise e interpretação dos dados, redação do artigo e aprovou a versão final a ser publicada. P. F. B. Almeida participou da revisão da literatura, redação do artigo, organização, análise e interpretação dos dados. C. O. Paixão participou da elaboração do projeto de pesquisa, organização, análise, interpretação dos dados e revisão do artigo. P. G. Bicalho participou da redação do artigo e realizou uma revisão crítica relevante do conteúdo intelectual. L. S. P. Errico participou da redação do artigo, organização, análise e interpretação dos dados, realizou uma revisão crítica relevante do conteúdo intelectual e aprovou a versão final a ser publicada.

\section{Referências}

1. Say L, Chou D, Gemmill A, Tunçalp O, Moller AB, Daniels J, et al. Global causes of maternal death: a WHO systematic analysis. Lancet Glob Health 2014; 2:e323-33.

2. World Health Organization. Trends in maternal mortality: 1990 to 2010. Geneva: World Health Organization; 2012.

3. Lozano R, Wang H, Foreman KJ, Rajaratnam JK, Naghavi M, Marcus JR, et al. Progress towards Millennium Development Goals 4 and 5 on maternal and child mortality: an updated systematic analysis. Lancet 2011; 378:1139-65.

4. Instituto de Pesquisa Econômica Aplicada. Objetivos de Desenvolvimento do Milênio: relatório nacional de acompanhamento. Brasília: Instituto de Pesquisa Econômica Aplicada; 2014.

5. Kassebaum NJ, Bertozzi-Villa A, Coggeshall MS, Shackelford KA, Steiner C, Heuton KR, et al. Glob$\mathrm{al}$, regional, and national levels and causes of maternal mortality during 1990-2013: a systematic analysis for the Global Burden of Disease Study 2013. Lancet 2014; 384:980-1004.

6. Souza KV, Almeida MRCB, Soares VMN. Perfil da mortalidade materna por aborto no Paraná: 20032005. Esc Anna Nery Rev Enferm 2008; 12:741-9.

7. Gerdts C, Vohra D, Ahern J. Measuring unsafe abortion-related mortality: a systematic review of the existing methods. PLoS One 2013; 8:e53346.

8. Departamento de Análise de Situação em Saúde, Secretaria de Vigilância em Saúde, Ministério da Saúde. Saúde Brasil 2012: uma análise da situação de saúde e dos 40 anos do Programa Nacional de Imunizações. Brasília: Ministério da Saúde; 2013.

9. Departamento de Ciência e Tecnologia, Secretaria de Ciência, Tecnologia e Insumos Estratégicos, Ministério da Saúde. Aborto e saúde pública no Brasil: 20 anos. Brasília: Ministério da Saúde; 2009.

10. World Health Organization. Unsafe abortion: global and regional estimates of the incidence of unsafe abortion and associated mortality in 2008. $6^{\text {th }}$ Ed. Geneva: World Health Organization; 2011.

11. Shah I, Ahman E. Unsafe abortion: global and regional incidence, trens, consequences and challenges. J Obstet Gynaecol Can 2009; 31:1149-58.

12. Jewkes R, Brown H, Dickson-Tetteh K, Levin J, Rees H. Prevalence of morbidity associated with abortion before and after legalisation in South Africa. BMJ 2002; 324:1252-3.

13. Jewkes R, Rees H, Dramatic decline in abortion mortality due to the choice on termination of pregnancy Act. S Afr Med J 2005; 95:250. 
14. Kulczycki A. Abortion in Latin America: changes in practice, growing conflict, and recent policy developments. Stud Fam Plann 2011; 42:199-220.

15. Matias JP, Parpinelli MA, Nunes MKV, Surita FGC, Cecatti JG. Comparação entre dois métodos para investigação da mortalidade materna em município do Sudeste brasileiro. Rev Bras Ginecol Obstet 2009; 31:559-65.

16. Domingos SRF, Merighi MAB. O aborto como causa de mortalidade materna: um pensar para o cuidado de enfermagem. Esc Anna Nery Rev Enferm 2010; 14:177-81.

17. Departamento de Análise de Situação em Saúde, Secretaria de Vigilância em Saúde, Ministério da Saúde. Guia de vigilância epidemiológica do óbito materno. Brasília: Ministério da Saúde; 2009.

18. Gomes FA, Mamede MV, Costa Junior ML, Nakano AMS. Morte materna mascarada: um caminho para sua identificação. Acta Paul Enferm 2006; 19:387-93

19. Laurenti R, Jorge MHPM, Gotlieb SLD. A mortalidade materna nas capitais brasileiras: algumas características e estimativa de um fator de ajuste. Rev Bras Epidemiol 2004; 7:449-60.

20. Rezende EM, Sampaio IBM, Ishitani LH, Martins EF, Vilella LCM. Mortalidade de idosos com desnutrição em Belo Horizonte, Minas Gerais, Brasil: uma análise multidimensional sob o enfoque de causas múltiplas de morte. Cad Saúde Pública 2010; 26:1109-21.

21. Santo AH. Causas múltiplas de morte: formas de apresentação e métodos de análise [Tese de Doutorado]. São Paulo: Faculdade de Saúde Pública, Universidade de São Paulo; 1988.

22. Ministério da Saúde. Manual de instruções para o preenchimento da declaração de óbito. 3a Ed. Brasília: Ministério da Saúde; 2001.

23. Organização Mundial da Saúde. Classificação estatística internacional de doenças e problemas relacionados à saúde, 10a revisão. São Paulo: Centro Colaborador da OMS para a Classificação de Doenças em Português; 1995.

24. Rede Interagencial de Informação para a Saúde. Indicadores básicos para a saúde no Brasil: conceitos e aplicações. 2a Ed. Brasília: Organização Pan-Americana da Saúde; 2008.

25. Senado Federal. Decreto-lei no $2.848,7$ de dezembro de 1940. http://legis.senado.gov.br/legislacao/ ListaPublicacoes.action? $\mathrm{id}=102343$ (acessado em 21/Jul/2015).

26. Organização Mundial da Saúde. Abortamento seguro: orientação técnica e de políticas para sistemas de saúde. 2a Ed. Genebra: Organização Mundial da Saúde; 2013.
27. Departamento de Ações Programáticas Estratégicas, Secretaria de Atenção à Saúde, Ministério da Saúde. Política Nacional de Atenção Integral à Saúde da Mulher: princípios e diretrizes. Brasília: Ministério da Saúde; 2004.

28. Secretaria de Estado da Saúde de Minas Gerais. Programa Viva Vida - construção dos comitês de investigação e mortalidade materna fetal e infantil. Belo Horizonte: Secretaria de Estado da Saúde de Minas Gerais; 2004.

29. Departamento de Ações Programáticas Estratégicas, Secretaria de Atenção à Saúde, Ministério da Saúde. Atenção humanizada ao abortamento: norma técnica. 2a Ed. Brasília: Ministério da Saúde; 2011. (Série A. Normas e Manuais Técnicos).

30. Ministério da Saúde. Portaria no 1.459, de 24 de junho de 2011. Institui, no âmbito do Sistema Único de Saúde - SUS, a Rede Cegonha. Diário Oficial da União 2011; 27 jun.

31. Secretaria Executiva, Ministério da Saúde. Programa humanização do parto: humanização no prénatal e nascimento. Brasília: Ministério da Saúde; 2000.

32. Malta DC, Duarte EC. Causas de mortes evitáveis por ações efetivas dos serviços de saúde: uma revisão da literatura. Ciênc Saúde Coletiva 2007; 12:765-76.

33. Drezett J. Mortalidade materna no Brasil. Insucesso no cumprimento do quinto Objetivo de Desenvolvimento do Milênio. Reprod Clim 2013; 28: 89-91.

34. Menezes G, Aquino EML. Pesquisa sobre o aborto no Brasil: avanços e desafios para o campo da saúde coletiva. Cad Saúde Pública 2009; 25 Suppl 2:S193-204.

35. Cecatti JG, Guerra GVQL, Souza MH, Menezes GMS. Aborto no Brasil: um enfoque demográfico. Rev Bras Ginecol Obstet 2010; 32:105-11.

36. Faria DR, Sousa RC, Costa TJNM, Leite ICG. Mortalidade materna em cidade-polo de assistência na Região Sudeste: tendência temporal e determinantes sociais. Rev Med Minas Gerais 2012; 22:18-25.

37. Machado CJ, Lobato ACL, Melo VH, Guimarães MDC. Perdas fetais espontâneas e voluntárias no Brasil em 1999-2000: um estudo de fatores associados. Rev Bras Epidemiol 2013; 16:18-29.

38. Coutinho T, Coutinho CM, Coutinho LM. Gravidez ectópica em cicatriz de cesárea: uma complicação emergente. Femina 2014; 42:19-26.

39. Dias JMG, Oliveira APS, Cipolotti R, Monteiro KKSM, Pereira RO. Mortalidade materna. Rev Med Minas Gerais 2015; 25:173-9. 


\section{Abstract}

This study aimed to analyze trends in maternal mortality from abortion in Minas Gerais State, Brazil, from 2000 to 2011, addressing multiple causes of death. The analysis focused on women's social and demographic characteristics, year, location, and underlying and associated causes of death. Maternal mortality ratio (MMR) and abortion-specific ratio were calculated for each year, as well as Spearman's correlation coefficient ( $p<$ 0.05 ), to assess the trend in ratios during the study period. One hundred and eighty-three deaths from abortion were recorded, accounting for $15 \%$ of maternal deaths, and MMR remained stable. The ratio between multiple causes and underlying cause of death from abortion was 1.38. Use of the multiple-causes method proved effective for lending greater visibility to abortion. Problems deriving from the illegality of abortion in Brazil favor the appearance of causes that mask maternal deaths, in addition to their underreporting. Inter-sector actions are thus necessary to define strategies for reducing social inequalities and improving the quality of services for women.

Maternal Mortality; Abortion; Multiple Cause of Death

\section{Resumen}

Este estudio tuvo por objetivo analizar la evolución de la mortalidad materna por aborto en Minas Gerais, Brasil, durante el período de 2000 a 2011, desde el enfoque de las causas múltiples de muerte. Se estudiaron las características sociodemográficas de las mujeres, año, lugar y causas básicas y asociadas de óbito. Se calculó la razón de muerte materna (RMM) general y especifica por aborto durante cada año y el coeficiente de correlación de Spearman $(p<0,05)$, para evaluar la evolución de las razones durante el período. Se registraron 183 óbitos por aborto, un 15\% de los óbitos maternos, y la RMM por aborto se mantuvo estable. La razón causa múltiple y causa básica de óbito por aborto fue de 1,38. El uso del método de análisis de causas múltiples se mostró eficaz para dar mayor visibilidad al aborto. Los problemas provenientes de la ilegalidad de la práctica de abortos favorecen la aparición de causas que enmascaran muertes maternas y su subnotificación. Por ello, se necesitan acciones intersectoriales para la definición de estrategias, con el fin de reducir las desigualdades sociales y mejorar la calidad de los servicios de atención a la mujer.

Mortalidad Materna; Aborto;

Causas Múltiples de Muerte

Recebido em 14/Ago/2015

Versão final reapresentada em 30/Mar/2016

Aprovado em 18/Abr/2016 